\title{
The Detection, Outcome, and Presentation of Twin-Twin Transfusion Syndrome in Monochorionic Diamniotic Twin Pregnancies Followed with a Protocol of Fortnightly Ultrasound Examination
}

\author{
Isabel Couck ${ }^{a}$ Sophie Ponnet ${ }^{a}$ Liesbeth Thewissen ${ }^{b}$ Francesca Russo $^{a}$ \\ Jan Deprest ${ }^{a, c, d}$ Luc De Catte ${ }^{a, c}$ Roland Devlieger ${ }^{a, c}$ Liesbeth Lewi ${ }^{a, c}$ \\ ${ }^{a}$ Department of Obstetrics and Gynecology, University Hospitals Leuven, Leuven, Belgium; ${ }^{b}$ Neonatal Intensive \\ Care Unit, University Hospitals Leuven, Leuven, Belgium; ' ${ }^{\circ}$ Department of Development and Regeneration, Cluster \\ Woman and Child, Biomedical Sciences, KU Leuven, Leuven, Belgium; IInstitute for Women's Health University \\ College London Hospital, London, UK
}

\section{Contribution}

- What is already known about the topic?

- If detected in time, fetoscopic laser surgery of the vascular anastomoses improves perinatal outcomes.

- Survival rates after fetoscopic laser surgery are better in stage I-II twin-twin transfusion syndrome than in stage III-IV.

\section{What does this study add?}

- In $90 \%$ of cases, a protocol of fortnightly ultrasound scan detects twin-twin transfusion in time. In $10 \%$, twin-twin transfusion syndrome (TTTS) presents with fetal demise, especially before 16 weeks or after 26 weeks.

- The survival of TTTS in an unselected cohort is lower than that after fetoscopic laser surgery.

- In stage III-IV, abnormal Doppler measurements precede the diagnosis of TTTSs, suggesting that the staging system does not reflect a progressive worsening of the disease, and more frequent follow-up may not result in an earlier stage at diagnosis.

\section{Keywords}

Monochorionic twin pregnancy - Ultrasound .

Fetofetal transfusion syndrome · Fetoscopy · Laser .

Detection · Outcome

\begin{abstract}
Background: Evidence to support a fortnightly scan protocol for monochorionic diamniotic (MCDA) pregnancies to detect twin-twin transfusion syndrome (TTS) is scarce. Also, TTSrelated mortality in an unselected cohort is not well documented. Finally, common knowledge suggests that a more
\end{abstract}

Correspondence to:

Liesbeth Lewi, liesbeth.lewi@uzleuven.be 
frequent follow-up may pick up the disease at a milder stage, but little is known on the ultrasound findings before the diagnosis. Objectives: We examine if a fortnightly ultrasound scan from 16 weeks onward detects TTS in time. Also, we document the outcomes in a large unselected cohort of MCDA twins and examine the ultrasound findings within 14 days before diagnosis. Methods: Retrospective cohort of 675 MCDA twin pregnancies followed with a fortnightly scan protocol from 16 weeks onward. Timely detection of TTTS was defined as before fetal demise (stage $\mathrm{V}$ ), ruptured membranes, or a dilated cervix. We compared the ultrasound findings before the diagnosis between stage I-II and stage III-IV. Results: A total of 82/675 (12\%) pregnancies developed TTS, of which $74 / 82$ (90\%) were detected in time. In 8/82 (10\%), TाTS was diagnosed in stage V: 5 before 16 weeks and 2 after 26 weeks. Fetoscopic laser photocoagulation (FLP) of the placental anastomoses was performed in 48/82 (59\%). The survival of TTTS in the entire cohort was 105/164 (64\%). In contrast, survival after FLP was 77/96 (80\%). In 16/19 (84\%) of stage III-IV TTTS, abnormal Doppler findings preceded the diagnosis of TTTS. Conclusions: A scheme of fortnightly ultrasound scans from 16 weeks onward detects 9 out of ten TTTS pregnancies in time. Most stage $V$ cases presented outside the typical time window of 16 and 26 weeks. Survival rates after FLP underestimate the mortality of TTTS. Most stage III-IV cases have abnormal Doppler findings before the diagnosis of TTS.

๑) 2021 S. Karger AG, Basel

\section{Introduction}

Twin-twin transfusion syndrome (TTTS) is the most severe complication in monochorionic diamniotic (MCDA) twin pregnancies. TTTS occurs in about one in ten pregnancies, usually between 16 and 26 weeks [1]. Untreated, it is associated with high mortality and morbidity rates [2]. Fetoscopic laser photocoagulation (FLP) of the placental anastomoses is currently the best treatment. Compared to amnioreduction, FLP is associated with higher perinatal survival rates, birth at a more advanced gestational age, and better neurodevelopmental outcomes [3]. In selected cases, such as a major anomaly in one twin, an inaccessible vascular equator, or patient preference, selective reduction may be an alternative to FLP to save one twin [4]. Invasive fetal therapy is only useful in the absence of intrauterine fetal demise (IUFD), with intact membranes and before cervical dilation.

Increased surveillance may help diagnose TTTS in time. Therefore, international guidelines recommend fortnightly sonographic evaluations for all MCDA twin pregnancies, starting at 16 weeks and continuing until birth [5-8]. However, there are no comparative studies such as fortnightly scheme's efficacy, and this recommendation is supported by a few cohort studies only [9-11]. Also, although the outcome after FLP is well documented with recent series reporting a $74 \%$ survival with at least one twin surviving in $88 \%$ [12], the outcome of TTTS in an unselected monochorionic twin population is not well known. Finally, common knowledge suggests that a more frequent follow-up may pick up the disease at an earlier stage, improving outcomes [13]. However, little is known on the ultrasound findings before the diagnosis.

The primary aim was to assess the proportion of TTTS pregnancies that is detected in time by a fortnightly ultrasound exam. We defined in time as before IUFD, ruptured membranes, and cervical dilation because we do not offer FLP under these circumstances. Second, we document the survival and neonatal outcome of TTTS in an unselected cohort of MCDA twin pregnancies followed from the first trimester and the ultrasound findings within 14 days of the diagnosis of TTTS.

\section{Methods}

\section{Study Population}

We performed a retrospective study of ongoing MCDA twin pregnancies diagnosed in the first trimester $(11+0-14+0$ weeks $)$ between January 2002 and September 2018. MCDA twin pregnancies are routinely referred to the twin clinic at the University Hospitals of Leuven (study center) for a detailed ultrasound scan at $11-14,16,20$, and $26-30$ weeks. In addition to these 4 examinations, patients are instructed to have a sonographic assessment at least every 2 weeks, either at the study center or the referring institution, to detect TTTS in a timely fashion as per recommendation [5-8]. Patients explicitly referred in the first trimester for invasive testing or because of an anomaly were not included. The same cohort was included in an earlier study on selective growth restriction and the impact of in vitro fertilization $[14,15]$.

The ultrasound scans were performed according to a well-defined protocol $[1,14]$ by experienced sonographers on Voluson E10/E8/E6/730 (GE Healthcare, Chicago, IL, USA). Ultrasound reports were made using the Astraia software (Astraia software gmbh, Munich, Germany). All patients were informed about TTTS and its warning signals at the first visit. When we detected a discordance in growth, amniotic fluid, or Doppler findings, we increased the frequency of ultrasound evaluations to weekly or twice-weekly exams depending on the severity and the gestational age.

Patients with TTTS were offered FLP as first-line treatment. Selective reduction by umbilical cord coagulation or intra-fetal radiofrequency ablation was offered as an alternative, especially if one twin had an anomaly, was deemed to have a poor prognosis, or if FLP was technically not feasible. Patients could also opt for termination of pregnancy if TTTS presented before viability. After 28 weeks, TTTS was mostly managed either by an elective birth or by amnioreduction. The Ethics Committee of the University Hospital Leuven approved the study (S63561). 


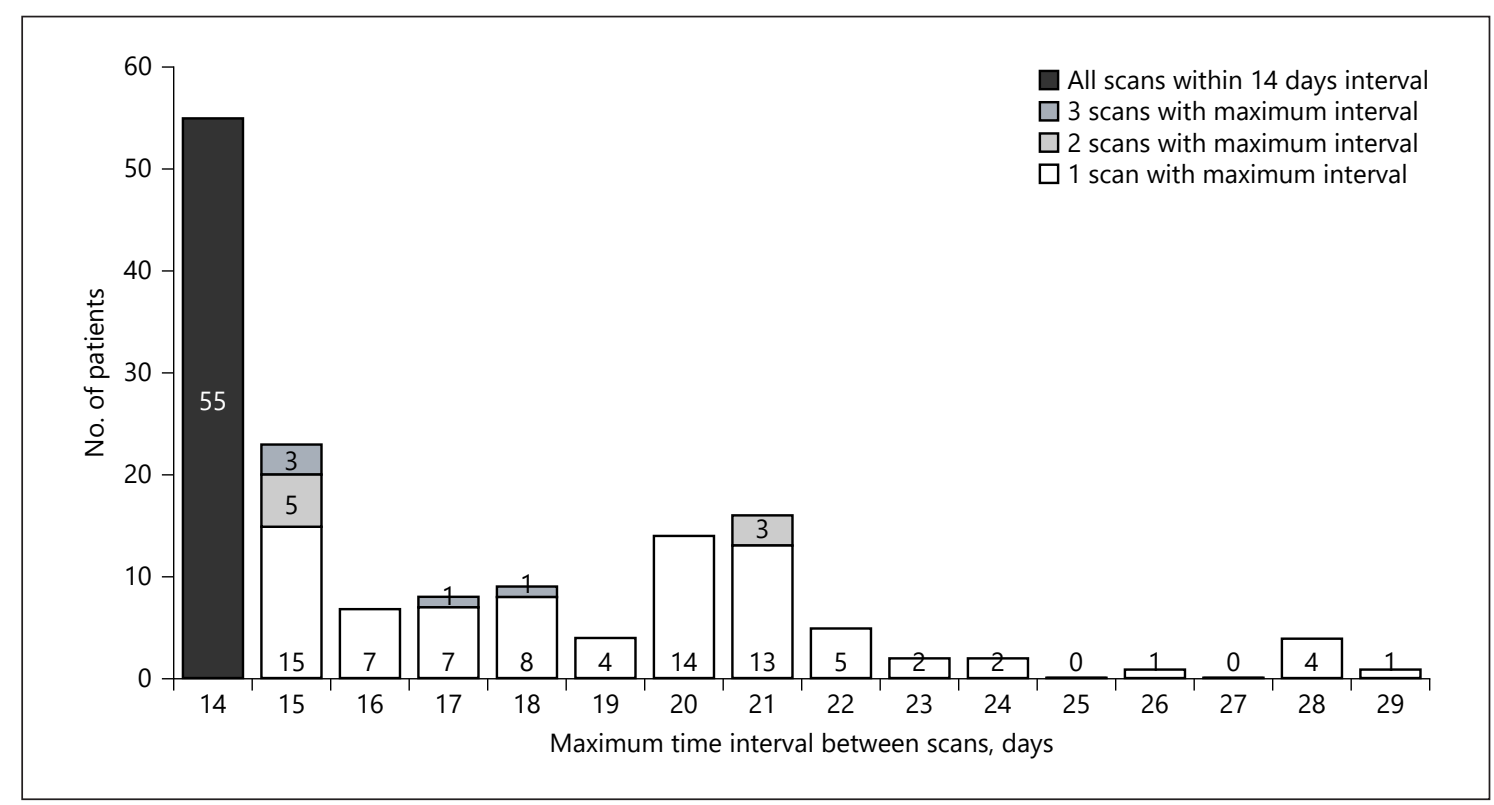

Fig. 1. Illustration of the patients who had their ultrasound exams strictly within the recommended 14-day interval (black bar; 55 of 151), as well as the maximum interval in between scans for the remainder of patients, for example, 15 of 151 patients had one exam with a 15-day interval, 5 of 151 patients had 2 exams with a 15-day interval, and 3 of 151 patients had 3 exams with a 15-day interval.

\section{Definitions}

TTTS was diagnosed by oligohydramnios and a small or empty bladder in the donor and polyhydramnios with a distended bladder in the recipient. Oligohydramnios was defined as a deepest vertical pocket (DVP) less than $2 \mathrm{~cm}$ [3]. Polyhydramnios was defined as a DVP more than $6 \mathrm{~cm}$ before 16 weeks [16], more than 8 $\mathrm{cm}$ before 20 weeks, and more than $10 \mathrm{~cm}$ after 20 weeks [3]. TTTS severity was assessed based on the Quintero staging system [17]. Timely detection of TTTS was defined as a diagnosis before IUFD, ruptured membranes, or dilated cervix.

Survival refers to fetal and neonatal survival up to 28 days of life per total number of included twin fetuses in the first trimester. Fetal loss included IUFD, miscarriage, and termination of pregnancy. Neonatal demise refers to the number of deaths within the first 28 days per total number of live-born babies. Severe neonatal morbidity was defined as either the occurrence of chronic lung disease (defined as oxygen dependency at 36 weeks), patent ductus arteriosus needing surgical treatment, necrotizing enterocolitis grade II or higher, retinopathy of prematurity stage III or higher, ischemic limb injury and amniotic band syndrome, and severe cerebral injury. Severe cerebral injury was defined as intraventricular hemorrhage grade III or higher, cystic periventricular leukomalacia grade II or higher, ventricular dilatation greater than the 97th percentile, porencephalic or parenchymal cysts, or other severe cerebral lesions associated with adverse neurological outcome. Neonatal brain scans were not always performed in infants after 32 weeks. In these cases, we collected the results from the fetal brain magnetic resonance imaging (MRI), which we have been offering to all TTTS pregnancies at 28-32 weeks from 2010 onward.

Detection, Outcome, and Presentation of TTTS

\section{Data Collection}

Ultrasound data were retrieved from the Astraia database. Pregnancy and neonatal outcome data were collected after birth from the electronic clinical records. If not present, they were requested from the referring physicians.

\section{Analyses of Primary and Secondary Outcomes}

The primary outcome is the proportion of TTTS pregnancies detected in a timely fashion. The secondary outcomes are the fetalneonatal survival and neonatal morbidity according to the management strategy, and the findings on the last scan prior to the diagnosis of TTTS in the subpopulation of patients that received this scan in our center.

\section{Results}

During the study period, $678 \mathrm{MCDA}$ pregnancies were followed from the first trimester onward. Three patients were lost to follow-up, which left 675 in the analysis. The average maternal age was 30 years, and 316/675 (47\%) were nulliparous. In 573/675 (85\%), the pregnancy was conceived spontaneously. The compliance with the 16, 20, and 30 week's exam at the study center was $613 / 637$ (96\%), $564 / 583$ (97\%), and 463/544 (85\%), respectively [14]. Of the $154(23 \%)$ patients, who initially booked for antenatal care in our center, the median scan interval was 14 days (range 1-29 days). Excluding the 3 patients that already 
Table 1. The clinical characteristics of stage V TTTS diagnosed before 16 weeks

\begin{tabular}{|c|c|c|c|c|c|}
\hline & Case 1 & Case 2 & Case 3 & Case 4 & Case 5 \\
\hline GA diagnosis TTTS & $15+3$ wks & $15+3$ wks & $15+3 \mathrm{wks}$ & $15+4 \mathrm{wks}$ & $15+5$ wks \\
\hline Findings & Double IUFD & Double IUFD & $\begin{array}{l}\text { IUFD donor } \\
\text { MCA recipient }>1.5 \\
\text { MoM } \\
\text { IUFD recipient } 15+6 \text { wks }\end{array}$ & $\begin{array}{l}\text { IUFD donor } \\
\text { Acrania recipient }\end{array}$ & $\begin{array}{l}\text { IUFD donor } \\
\text { MCA recipient }>1.5 \\
\text { MoM } \\
\text { IUT } 15+5 \text { wks - Hb } 5.5 \\
\text { g/dL* }\end{array}$ \\
\hline DVP donor/recipient & DVP $0 / 6.5 \mathrm{~cm}$ & DVP $2.0 / 7.3 \mathrm{~cm}$ & DVP $0 / 7.7 \mathrm{~cm}$ & DVP $0 / 6.0 \mathrm{~cm}$ & DVP $1.2 / 8.5 \mathrm{~cm}$ \\
\hline $\begin{array}{l}\text { Last scan before IUFD } \\
\text { Findings }\end{array}$ & $\begin{array}{l}15+0 \text { wks } \\
\text { Abnormal flow } \\
\text { smaller } \\
\text { AEDF UA } \\
\text { Negative a-wave DV } \\
\text { Empty/full bladder } \\
\text { DVP } 2.1 / 5.0 \mathrm{~cm} \\
\text { Amnion-chorion } \\
\text { separated }\end{array}$ & $\begin{array}{l}\text { 14+6 wks } \\
\text { Abnormal flow smaller } \\
\text { AEDF UA } \\
\text { Negative a-wave DV } \\
\text { Empty/full bladder } \\
\text { DVP } 2.1 / 5.0 \mathrm{~cm} \\
\text { Amnion-chorion } \\
\text { separated } \\
\text { Coarctation small twin }\end{array}$ & $\begin{array}{l}14+3 \text { wks } \\
\text { Abnormal flow smaller } \\
\text { AEDF UA } \\
\text { DVP } 2.8 / 7.4 \mathrm{~cm} \\
\text { Amnion-chorion } \\
\text { separated }\end{array}$ & $\begin{array}{l}14+0 \text { wks } \\
\text { Empty/full bladder } \\
\text { DVP decreased/ } \\
\text { increased }\end{array}$ & $\begin{array}{l}12+2 \text { wks } \\
\text { See below }\end{array}$ \\
\hline First-trimester scan & $14+0 \mathrm{wks}$ & $12+6$ wks & $12+4$ wks & $13+3 \mathrm{wks}$ & $12+2 \mathrm{wks}$ \\
\hline Findings & $\begin{array}{l}22 \% \text { CRL discordance } \\
\text { Empty/full bladder } \\
\text { DVP } 1.8 / 4.7 \mathrm{~cm} \\
\text { SUA smaller twin } \\
\text { NT } 1.2 / 2.9 \mathrm{~mm} \\
\text { Both twins normal DV }\end{array}$ & $\begin{array}{l}\text { 23\% CRL discordance } \\
\text { Empty/full bladder } \\
\text { DVP } 2.3 / 4.1 \mathrm{~cm} \\
\text { SUA smaller twin } \\
\text { NT } 1.3 / 3.8 \mathrm{~mm} \\
\text { Both twins negative DV }\end{array}$ & $\begin{array}{l}\text { 21\% CRL discordance } \\
\text { Empty/full bladder } \\
\text { DVP increased/ } \\
\text { decreased } \\
\text { NT } 0.8 / 2.8 \mathrm{~mm} \\
\text { Both twins normal DV }\end{array}$ & $\begin{array}{l}\text { Acrania } 1 \text { twin } \\
\text { Both bladders present } \\
\text { DVP normal/ } \\
\text { decreased } \\
\text { NT } 1.0 \mathrm{~mm}\end{array}$ & $\begin{array}{l}\text { 5\% CRL discordance } \\
\text { Both bladders visible } \\
\text { Both twins normal } \\
\text { fluid } \\
\text { NT } 0.8 / 2.3 \mathrm{~mm} \\
\text { Both twins normal DV }\end{array}$ \\
\hline Management & PG induction & PG induction & PG induction & TOP & $\begin{array}{l}\text { Birth } 38+5 \text { wks } 3,890 \mathrm{~g} \\
\text { Normal MRI } 30 \text { wks }\end{array}$ \\
\hline
\end{tabular}

TTTS, twin-twin transfusion syndrome; DVP, deepest vertical pocket; IUFD, intrauterine fetal demise; wks, weeks; AEDF, absent end-diastolic flow; UA, umbilical artery; DV, ductus venosus; CRL, crown-rump length; SUA, single umbilical artery; NT, nuchal translucency; PG, prostaglandin; MCA, middle cerebral artery; TOP, termination of pregnancy; IUT, intrauterine transfusion; Hb, hemoglobin; g, grams; MRI, magnetic resonance imaging. * Using a 22 -gauge needle, $8 \mathrm{~mL}$ was transfused in the umbilical vein at the placental insertion and $4 \mathrm{~mL}$ intraperitoneal.

developed TTTS by 16 weeks, the scan interval never exceeded 21 days in 136 of 151 (90\%) patients (Fig. 1).

TTTS occurred in 82 of 675 (12\%) pregnancies. In 11 of $82(13 \%)$, TTTS was diagnosed before 16 weeks and 12 of $82(15 \%)$ after 26 weeks. The survival rate was 105/164 (64\%), with the survival of at least one twin in $65 / 82(79 \%)$. Severe neonatal morbidity occurred in 10/105 (10\%), and severe cerebral injury was diagnosed in $6 / 105(6 \%)$. In $86 / 105$ (82\%) infants, brain scans were performed in the neonatal period. From the remaining 19 infants, all born between 33 and 39 weeks, information on cerebral injury was obtained from the third-trimester MRI scan.

In $8 / 82(10 \%)$ cases, the diagnosis of TTTS was made after IUFD of one or both twins (stage V). None presented with ruptured membranes or a dilated cervix at diagnosis. The clinical characteristics of stage $\mathrm{V}$ diagnoses are summarized in Table 1 (case 1-5) and Table 2 (case 6-8).
In 5/8 stage $\mathrm{V}$ patients, TTTS was diagnosed before 16 weeks (case 1-5). At the first-trimester scan, 4 had discordant amniotic fluid (case 1-4), 3 had a crown-rump length (CRL) discordance of more than $20 \%$ (case 1-3), and in another case, acrania was diagnosed in the larger twin (case 4). Therefore, these 4 pregnancies (case 1-4) had additional ultrasound scans 3 to 10 days before the demise.

In $3 / 8$ stage $\mathrm{V}$ patients, TTTS was diagnosed after viability (case 6-8). One double IUFD occurred at 24 weeks in a patient who was followed alternating with the referring physician and did not attend her ultrasound appointments between 16 and 24 weeks (case 6). This same patient also had a stage V TTTS at 15 weeks in a subsequent pregnancy (case 5). One single IUFD (case 7) occurred at 26 weeks, 2 weeks after a normal exam, and a double IUFD (case 8 ) occurred at 27 weeks, 8 days after the ultrasound scan showed mild discordant fluid. 
Table 2. The clinical characteristics of stage V TTTS diagnosed after viability

\begin{tabular}{|c|c|c|c|}
\hline & Case 6 & Case 7 & Case 8 \\
\hline GA diagnosis & $24+0 \mathrm{wks}$ & $26+3 \mathrm{wks}$ & $27+3$ wks \\
\hline DVP donor/recipient & Polyanhydramnios $\dagger$ & DVP $1.7 / 12.8 \mathrm{~cm}$ & DVP $0 / 15 \mathrm{~cm}$ \\
\hline Findings & $\begin{array}{l}\text { Both twins normal flow } \\
\text { Both bladders visible } \\
\text { Both twins normal fluid } \\
\text { Both cords velamentous }\end{array}$ & Normal ultrasound scan $\dagger$ & $\begin{array}{l}\text { Both twins normal flow } \\
\text { Both bladders visible } \\
\text { DVP } 3.7 / 6.0 \mathrm{~cm}\end{array}$ \\
\hline First-trimester scan & $12+1$ wks & $13+0$ wks & $12+2$ wks \\
\hline Management & PG induction & $\begin{array}{l}\text { Birth } 37+1 \text { wks } 2,510 \mathrm{~g} \\
\text { Normal MRI } 32 \text { wks }\end{array}$ & PG induction \\
\hline
\end{tabular}

TTTS, twin-twin transfusion syndrome; DVP, deepest vertical pocket; IUFD, intrauterine fetal demise; wks, weeks; DV, ductus venosus; CRL, crown-rump length; NT, nuchal translucency; PG, prostaglandin; MCA, middle cerebral artery; MRI, magnetic resonance imaging. ${ }^{\dagger}$ Ultrasound scan performed by referring physician.

Table 3. The clinical details, management, and outcome of TTTS pregnancies. Data are expressed as median (range) and proportion (\%)

\begin{tabular}{|c|c|c|c|c|}
\hline TTTS I & $20 / 82(24 \%)$ & $15 / 48(31 \%)$ & $0 / 15(0 \%)$ & $4 / 9(44.5 \%)$ \\
\hline TTTS II & $19 / 82(23 \%)$ & $13 / 48(17 \%)$ & $5 / 15(33 \%)$ & $1 / 9(11 \%)$ \\
\hline TTTS III & $33 / 82(40 \%)$ & $19 / 48(40 \%)$ & $9 / 15(60 \%)$ & $4 / 9(44.5 \%)$ \\
\hline GA treatment/diagnosis ${ }^{\#}$ & $18+3(14+1-32+4) \mathrm{wks}$ & $18+3(14+1-28+0) \mathrm{wks}$ & $17+3(15+0-28+0) \mathrm{wks}$ & $29+4(14+5-32+4)$ wks \\
\hline GA birth & $33+3(25+0-40+0)$ wks & $33+3(25+0-38+3)$ wks & $38+1(26+6-40+0)$ wks & $30+3(28+4-35+6) \mathrm{wks}$ \\
\hline Birth by C-section & $46 / 67(69 \%)$ & $33 / 46(72 \%)$ & $4 / 10(40 \%)$ & $8 / 9(89 \%)$ \\
\hline Birth weight & $1,673(630-3,890) \mathrm{g}$ & $1,680(630-3,460) \mathrm{g}$ & $2,878(910-3,795) \mathrm{g}$ & $1,300(930-2,050) \mathrm{g}$ \\
\hline Fetal loss & $57 / 164(35 \%)$ & $18 / 96(19 \%)^{\ddagger}$ & $20 / 30(67 \%)^{\S}$ & $1 / 18(6 \%)$ \\
\hline Survival of at least one twin & $65 / 82(79 \%)$ & $45 / 48(94 \%)$ & $9 / 15(60 \%)$ & 9/9 (100\%) \\
\hline Severe neonatal morbidity & $10 / 105(10 \%)$ & $6 / 77(8 \%)$ & $2 / 9(22 \%)$ & $2 / 17(12 \%)$ \\
\hline Severe cerebral injury & $6 / 105(6 \%)$ & $4 / 77(5 \%)$ & $0 / 9(0 \%)$ & $2 / 17(12 \%)$ \\
\hline
\end{tabular}

TTTS, twin-twin transfusion syndrome; wks, weeks; C-section, caesarean section; TOP, termination of pregnancy. ${ }^{\dagger}$ Including the 8 stage $\mathrm{V}$ cases from Table 1 , and 2 patients with stage II TTTS who opted for TOP. ${ }^{9}$ Including only live-born infants after 24 weeks. ${ }^{\ddagger}$ Includes 1 TOP. ${ }^{\S}$ Includes 1 TOP and 1 miscarriage. " GA at first diagnosis for stage $\mathrm{V}$ and patients who opted for TOP or were managed expectantly. 
Table 4. The ultrasound findings before the diagnosis of TTTS in stage I-II and III-IV. Data are expressed as median (range) and proportion (\%)

\begin{tabular}{|c|c|c|c|}
\hline $\begin{array}{l}\text { Scan in study center } \leq 14 \text { days before TTTS diagnosis } \\
N=55\end{array}$ & $\begin{array}{l}\text { Diagnosis in stage I-II } \\
N=27\end{array}$ & $\begin{array}{l}\text { Diagnosis stage III-IV } \\
N=23\end{array}$ & $p$ value \\
\hline GA at first diagnosis of TTTS & $19+5(16+4-30+4)$ wks & $17+0(13+6-28+3) \mathrm{wks}$ & $<0.01$ \\
\hline Abnormal Dopplers on pre-TTTS scan ${ }^{\dagger}$ & $1 / 27(4 \%)$ & $16 / 19(84 \%)^{9}$ & $<0.0001$ \\
\hline Discordant growth on pre-TTTS scan & $8 / 27(30 \%)$ & $12 / 23(52 \%)$ & 0.10 \\
\hline Discordant amniotic fluid on pre-TTTS scan & $26 / 27(96 \%)$ & $22 / 23(96 \%)$ & 1.00 \\
\hline
\end{tabular}

TTTS, twin-twin transfusion syndrome. ${ }^{\dagger}$ Defined as absent or reversed end-diastolic flow in the umbilical artery or absent or reversed a-wave in the ductus venosus of one or both twins. ${ }^{9}$ Exclusion of 4 cases in which the pre-TTTS scan was the 1st trimester scan as umbilical artery Doppler was not measured; in 6 abnormal ductus venous in future recipient; in 6 abnormal umbilical artery in future donor; in 1 abnormal ductus in future donor; in 3, both future recipient and donor had abnormal Doppler findings. ${ }^{\ddagger}$ Defined as a crownrump length discordance of $\geq 10 \%$ in the 1 st trimester and an estimated fetal weight discordance of $\geq 20 \%$ after the 1 st trimester.

The survival in the 8 stage $\mathrm{V}$ cases was $2 / 16(12.5 \%)$ with the survival of at least one twin in $2 / 8(25 \%)$. One surviving twin underwent an intrauterine transfusion at 16 weeks. Both surviving twins had a normal fetal brain MRI at 30-32 weeks and were born at term.

In 74/82 (90\%), TTTS was diagnosed before IUFD of one or both twins. The clinical details, management, and outcome of TTTS pregnancies that were detected in a timely fashion are summarized in Table 3.

In 48/74 (65\%) patients, FLP was performed as a firstline intervention. The survival rate was $77 / 96(80 \%)$, with the survival of at least one twin in 45/48 (94\%). Four patients required an additional intervention: 3 selective reductions ( 2 recurrent TTTS and one severe cerebral injury) and one intrauterine transfusion/exchange transfusion for twin anemia polycythemia syndrome.

In 15/74 (20\%) patients, a primary selective reduction was performed for a discordant anomaly $(n=6)$, severe growth restriction $(n=4)$, or technical limitations to perform an FLP procedure $(n=5)$. In 9 of 15 pregnancies $(60 \%)$, selective reduction resulted in a surviving infant.

In $9 / 74$ (12\%) patients, no surgical intervention was performed, resulting in a survival rate of $17 / 18(94 \%)$ and the survival of at least one twin in $9 / 9$ (100\%). Four patients were electively delivered between 30 and 33 weeks. Two other patients underwent an amnioreduction at 28 and 30 weeks. Another 3 patients were managed expectantly. One case was diagnosed at 15 weeks (stage III), the second at 16 weeks (stage II), and the third at 20 weeks in stage I. Because of technical limitations, we chose initially for conservative management in these 3 patients, which resulted in a spontaneous resolution, albeit with the unexpected IUFD of a former recipient at 26 weeks in the third case.
Finally, 2/74 (3\%) patients with stage II and III disease opted for termination as primary management. In one pregnancy, both twins had a congenital heart defect.

Two-thirds of TTTS pregnancies (55/82) had an ultrasound scan at the study center within 14 days of diagnosis with a median interval of 4 days. The ultrasound findings on the pre-TTTS scan of pregnancies presenting as mild (stage I and II) and severe (stage III and IV) TTTS are summarized in Table 4. Of the patients who presented as severe TTTS, the majority already had abnormal Doppler measurements before TTTS was diagnosed. There was no difference in staging and outcome between pregnancies that did or did not have their previous scan at the study center within 14 days of diagnosis (Table A1).

\section{Discussion}

This study demonstrates that a protocol of fortnightly sonographic follow-up detects 9 out of ten TTTS cases in a timely fashion. As such, TTTS was diagnosed in stage $\mathrm{V}$ in 8 of 675 (1\%) MCDA twins. This high rate of timely detection is in agreement with the results of Sueters et al. [9], who reported the timely detection of 4 TTTS cases in 23 patients using a similar schedule.

In our series, 5 of 8 stage $\mathrm{V}$ pregnancies presented before 16 weeks, mostly within 10 days of a previous exam. At the first-trimester scan, 4 had discordant amniotic fluid, 3 had a CRL discordance of more than $20 \%$, and one had a nuchal translucency discordance of $65 \%$. CRL and nuchal translucency discordance increase the risk of TTTS [18]. In a recent series reporting on 1,274 MCDA pregnancies, a CRL discordance of more than $20 \%$ was 
present in $1 \%$ of MCDA twins, but $70 \%$ were complicated by IUFD or needed FPL before 20 weeks [19]. Since FLP can only be performed after amnion-chorion fusion, these early transfusion imbalances may result in IUFD before FLP can be offered. At present, the benefit of FLP performed before 16 weeks remains uncertain [20]. Therefore, the clinical relevance and impact of detecting these early TTTS cases may be limited.

Two previously uncomplicated pregnancies suddenly developed polyhydramnios after 26 weeks and were diagnosed in stage $\mathrm{V}$. The exact mechanism of these acute, late presentations is unknown but may be related to fetal vessel thromboses, which is more common in monochorionic placentas [21]. Such transfusion imbalances due to the occlusion of an anastomosis will remain unpredictable.

In our series, $49 \%$ of TTTS patients took home both babies, which is lower than the $67 \%$ dual survival rate after FLP in this study, and the $62 \%$ dual survival reported in the recent literature [12]. In our series, only $48 / 82$ (59\%) of TTTS cases were treated with FPL, which explains why the actual mortality of TTTS is higher than what is reported after FPL.

Of the patients presenting as stage III-IV TTTS, most already had abnormal Doppler findings on their preTTTS scan. Therefore, these patients did not pass through stage I and II disease first. Severe TTTS does not appear to result from a delayed diagnosis but seems to represent a different presentation of the disease in which Doppler abnormalities precede a severe amniotic fluid discordance. Therefore, the Quintero's stages of TTTS should not be interpreted as a consecutive worsening of the disease, but rather as distinct presentations with different underlying pathophysiology. Also, as Doppler abnormalities occur before a severe amniotic fluid discordance, a more frequent follow-up will not necessarily prevent a severe stage at diagnosis. Similarly, other studies did not find a relationship between the follow-up interval and staging at first diagnosis $[10,11]$.

Our study's strength is that it reports on an unselected cohort of MCDA twin pregnancies followed from the first trimester with fortnightly follow-up. As a limitation, we do not have exact information on the fortnightly scheme's compliance in patients who were followed up alternating with the referring physician. Indeed, one such patient with stage V TTTS at 24 weeks did not attend her appointments after 16 weeks. In $90 \%$ of patients who booked at our center, the scan interval never exceeded 21 days. Also, scan attendance at 16,20 , and 30 weeks was 96,97 , and $85 \%$, respectively. In Belgium, ultrasound appointments are covered by national health insurance, and patients are keen to know how their twin pregnancy evolves. Therefore, we expect a similarly good adherence for patients who were followed alternating with their referring physician.

In conclusion, our study shows that a scheme of fortnightly ultrasound scans is effective in detecting TTTS in time. None presented with ruptured membranes or cervical dilation, and most stage $\mathrm{V}$ cases were diagnosed before 16 weeks or presented with acute TTTS after 26 weeks. Only about $60 \%$ of all TTTS cases were treated with FLP. Therefore, the mortality was higher than that reported after FLP. Finally, most patients had abnormal Doppler findings before the diagnosis of stage III-IV TTTS, suggesting that these pregnancies did not pass through stage I and III first.

\section{Acknowledgements}

We thank the physicians for referring their patients for alternating follow-up in our center.

\section{Statement of Ethics}

The research was conducted ethically in accordance with the World Medical Association Declaration of Helsinki. The study was approved by the Medical Ethics Committee of the University Hospital Leuven, Leuven, Belgium (S62385). The local Ethics Committee of the University Hospital Leuven agreed on using retrospective data collected by the therapeutic team without written informed consent of included patients.

\section{Conflict of Interest Statement}

The authors have no conflicts of interest to declare. Liesbeth Lewi and Jan Deprest are both Editorial Board Members of Fetal Diagnosis and Therapy.

\section{Funding Sources}

This study was funded by a grant from the Fund for Academic Research of the University Hospitals Leuven, Belgium; LL is the recipient of a grant of "Fonds voor Wetenschappelijk Onderzoek" (FWO grantnr: 1804718N).

\section{Author Contributions}

Study conception and design: Isabel Couck, Sophie Ponnet, and Liesbeth Lewi; data acquisition: all authors; analysis and data interpretation: Isabel Couck, Sophie Ponnet, and Liesbeth Lewi; drafting of the manuscript: Isabel Couck, Sophie Ponnet, and Liesbeth Lewi; and critical revision: all authors. Isabel Couck and Sophie Ponnet contributed equally to this paper. 


\section{Appendix}

Table A1. The clinical details and outcome of TTTS pregnancies in relation to the timing of the last ultrasound scan at the study center. Data are expressed as median (range) and proportion (\%)

\begin{tabular}{|c|c|c|c|}
\hline & $\begin{array}{l}\text { Scan in study center } \\
\leq 14 \text { days before } \\
\text { TTTS diagnosis } \\
N=55\end{array}$ & $\begin{array}{l}\text { Scan in study center } \\
>14 \text { days before } \\
\text { TTTS diagnosis } \\
N=27\end{array}$ & $p$ value \\
\hline GA at first diagnosis & $17+6(13+6-30+4) \mathrm{wks}$ & $19+3(15+5-32+4) \mathrm{wks}$ & 0.18 \\
\hline Interval to last scan before TTTS diagnosis & $4(1-14)$ days & $24(15-55)$ days & $<0.0001$ \\
\hline TTTS I-II & $27 / 55(49 \%)$ & $10 / 27(37 \%)$ & \\
\hline TTTS III-IV & $23 / 55(42 \%)$ & $14 / 27(52 \%)$ & 0.30 \\
\hline TTTS V & $5 / 55(9 \%)$ & $3 / 27(11 \%)$ & \\
\hline Survival of both twins & $26 / 55(47 \%)$ & $14 / 27(55 \%)$ & 0.70 \\
\hline Survival of one twin & $19 / 55(35 \%)$ & $6 / 27(22 \%)$ & 0.25 \\
\hline Survival of at least one twin & $45 / 55(82 \%)$ & $20 / 27(77 \%)$ & 0.42 \\
\hline
\end{tabular}

TTTS, twin-twin transfusion syndrome; GA, gestational age at diagnosis.

\section{References}

1 Lewi L, Jani J, Blickstein I, Huber A, Gucciardo L, Van Mieghem T, et al. The outcome of monochorionic diamniotic twin gestations in the era of invasive fetal therapy: a prospective cohort study. Am J Obstet Gynecol. 2008 Nov; 199(5): 514-8.

2 Urig MA, Clewell WH, Elliott JP. Twin-twin transfusion syndrome. Am J Obstet Gynecol. 1990 Nov; 163(5 Pt 1):1522-6.

3 Senat MV, Deprest J, Boulvain M, Paupe A, Winer N, Ville Y. Endoscopic laser surgery versus serial amnioreduction for severe twin-totwin transfusion syndrome. N Engl J Med. 2004 Jul 8;351(2):136-44.

4 Lewi L, Gratacos E, Ortibus E, Van Schoubroeck D, Carreras E, Higueras T, et al. Pregnancy and infant outcome of 80 consecutive cord coagulations in complicated monochorionic multiple pregnancies. Am J Obstet Gynecol. 2006 Mar; 194(3):782-9.

5 Society for Maternal-Fetal Medicine; Simpson LL. Twin-twin transfusion syndrome. Am J Obstet Gynecol. 2013 Jan;208(1):3-18.

6 Khalil A, Rodgers M, Baschat A, Bhide A, Grata$\cos \mathrm{E}$, Hecher K, et al. ISUOG practice guidelines: role of ultrasound in twin pregnancy. Ultrasound Obstet Gynecol. 2016 Feb;47(2):24763.

7 Management of monochorionic twin pregnancy. Green-top guideline no. 51. BJOG. 2017 Jan; 124(1):e1-e45.

8 NICE guideline [NG137]. Twin and triplet pregnancy. 2019
9 Sueters M, Middeldorp JM, Lopriore E, Oepkes D, Kanhai HH, Vandenbussche FP. Timely diagnosis of twin-to-twin transfusion syndrome in monochorionic twin pregnancies by biweekly sonography combined with patient instruction to report onset of symptoms. Ultrasound Obstet Gynecol. 2006 Oct;28(5):659-64.

10 McDonald R, Hodges R, Knight M, Teoh M, Edwards A, Neil P, et al. Optimal Interval between ultrasound scans for the detection of complications in monochorionic twins. Fetal Diagn Ther. 2017;41(3):197-201

11 Brock CO, Sibai BM, Soto EE, Moise KJ Jr, Johnson A, Blackwell SC, et al. Fortnightly surveillance of monochorionic diamniotic twins for twin to twin transfusion syndrome: compliance and effectiveness. Prenat Diagn. 2020 Aug 11.

12 Akkermans J, Peeters SH, Klumper FJ, Lopriore E, Middeldorp JM, Oepkes D. Twenty-five years of fetoscopic laser coagulation in twin-twin transfusion syndrome: a systematic review. Fetal Diagn Ther. 2015;38(4):241-53.

13 Bamberg C, Diehl W, Diemert A, Sehner S, Hecher K. Neither the differentiation between twin-twin transfusion syndrome stages I and II nor III and IV makes a difference regarding the probability of double survival after laser therapy. Ultrasound Obstet Gynecol. 2020 Sep 22.

14 Couck I, Ponnet S, Deprest J, Devlieger R, de Catte L, Lewi L. Outcome of selective intrauterine growth restriction in monochorionic twin pregnancies at 16,20 or 30 weeks according to the new consensus definition. Ultrasound Obstet Gynecol. 2020 Jan 16;56.
15 Couck I, Van Nylen L, Deprest J, Lewi L. Monochorionic twins after in-vitro fertilization: do they have poorer outcomes? A retrospective cohort study. Ultrasound Obstet Gynecol. 2020 Jan 7.

16 Dekoninck P, Deprest J, Lewi P, Richter J, Galjaard S, Van Keirsbilck J, et al. Gestational agespecific reference ranges for amniotic fluid assessment in monochorionic diamniotic twin pregnancies. Ultrasound Obstet Gynecol. 2013 Jun;41(6):649-52.

17 Quintero RA, Morales WJ, Allen MH, Bornick PW, Johnson PK, Kruger M. Staging of twintwin transfusion syndrome. J Perinatol. 1999 Dec;19(8 Pt 1):550-5.

18 Stagnati V, Zanardini C, Fichera A, Pagani G Quintero RA, Bellocco R, et al. Early prediction of twin-to-twin transfusion syndrome: systematic review and meta-analysis. Ultrasound $\mathrm{Ob}-$ stet Gynecol. 2017 May;49(5):573-82.

19 Litwinska E, Syngelaki A, Cimpoca B, Sapantzoglou I, Nicolaides KH. Intertwin discordance in fetal size at 11-13 weeks' gestation and pregnancy outcome. Ultrasound Obstet Gynecol. 2019 Nov 11.

20 D'Antonio F, Benlioglu C, Sileo FG, Thilaganathan B, Papageorghiou A, Bhide A, et al. Perinatal outcomes of twin pregnancies affected by early twin-twin transfusion syndrome: a systematic review and meta-analysis. Acta Obstet Gynecol Scand. 2020 Mar 11.

21 Sato Y, Benirschke K. Increased prevalence of fetal thrombi in monochorionic-twin placentas. Pediatrics. 2006 Jan;117(1):e113-7. 